\title{
Investigation of short-term disturbances of the solar wind using a tensor anisotropy method
}

\author{
S.K. Gerasimova, P.Yu. Gololobov*, V.G. Grigoryev, P.A. Krivoshapkin, G.F. \\ Krymsky \\ Yu.G. Shafer Institute of Cosmophysical Research and Aeronomy of SB RAS, 31 Lenin ave., \\ 677980, Yakutsk, Russia \\ E-mail: gpeter@ikfia.sbras.ru
}

In this work the dynamics of tensor anisotropy of cosmic rays during the passage of large-scale disturbances of the solar wind for the 22-24 solar cycles is studied. The information on the anisotropy was obtained using a global survey method based on the data of the worldwide neutron monitor network. For the analysis of the obtained results the data on the interplanetary magnetic field state and solar wind parameters were used.

The 34th International Cosmic Ray Conference,

30 July- 6 August, 2015

The Hague, The Netherlands

* Speaker. 


\section{Introduction}

The continuous observation of cosmic rays with neutron monitors has been carried out for more than half of a century. The world-wide neutron monitor network whose data is presented at the website of neutron monitor database $\mathrm{NMDB}^{1}$ registers cosmic ray primary particles with the threshold energies up to $14.1 \mathrm{GeV}$.

Spreading in the heliosphere the galactic cosmic rays are exposed to various modulating effects of the solar wind. In the earlier works for explanation of 1 and 2 angular moments of cosmic ray distribution in the interplanetary medium, the mechanisms of convective-diffusive and drift motion of particles, shielding of cosmic rays by a sectorial magnetic field $[1,2]$ and shear flow of the solar wind $[3,4]$ were mainly proposed.

Deviations of cosmic rays from the isotropic distribution in the directions of their motion are very small - less than one percent. However, their all-round study is quite justified because they give the information on dynamic properties of the solar wind. A small value of anisotropy requires the application of special methods for data processing and their complex analysis for its study. Such methods were developed in ShICRA at 1960-s: crossed telescopes, receiving vectors and global survey and. This methods are described in $[5,1]$.

In the work [6,7] using the methods of receiving vectors and crossed telescopes [1] during a period since 1971 to 2011 based on the data of underground complex of muon telescopes of st. Yakutsk ${ }^{2}$ and multidirectional muon telescopes of st. Nagoya ${ }^{3}$ the parameters of semidiurnal $R_{2}^{2}$ variation of cosmic ray intensity were calculated. The obtained results show a steady annual course of $R_{2}^{2}$ during all the period. Besides the semidiurnal variation, the second spherical of cosmic ray intensity also creates antisymmetric diurnal variations $R_{2}^{1}$. In the work [8] by the data of Nagoya muon telescopes the behaviour of this component was obtained. It turns out that the $R_{2}^{1}$ also has a seasonal change. In order to explain this seasonal changes of $R_{2}^{1}$ and $R_{2}^{2}$ the mechanisms of cosmic ray screening and solar wind shear flow were suggested.

In this work in addition to the earlier obtained results, the behavior of the tensor anisotropy components $\left(R_{2}^{1}\right.$ and $\left.R_{2}^{2}\right)$ during a short-term disturbances of solar wind was investigated. To study this short-term events special methods of data processing the global survey method is used. This method allows to determine hour to hour changes of the tensor anisotropy parameters. As experimental data we used the data of NMDB. We also used the data of interplanetary magnetic field intensity, solar wind speed, Dst-index which are provided by NASA database ${ }^{4}$ and computed coronal field synoptic charts of the Wilcox Solar Observatory ${ }^{5}$.

\section{Investigation method}

The cosmic ray distribution can be expanded into series of spherical functions which are solu-

\footnotetext{
${ }^{1}$ http://www.nmdb.eu

${ }^{2}$ www.ysn.ru/ipm

${ }^{3}$ www.stelab-nagoya-u.ac.jp/ste-www1/div3/muon

${ }^{4} \mathrm{http} / / /$ omniweb.gsfc.nasa.gov/form/dx1.html

${ }^{5} \mathrm{http}: / /$ wso.stanford.edu
} 
tions of Laplace's equation:

$$
J(\theta, \varphi)=\sum_{n=0}^{\infty} \sum_{m=0}^{n}\left(a_{n}^{m} \cos m \varphi+b_{n}^{m} \operatorname{sinm} \varphi\right) P_{n}^{m}(\sin \theta),
$$

where $\theta, \varphi$ are latitudinal and longitudinal angles in some coordinate system, $P_{n}^{m}$ is associated Legendre polynomials. The distribution of intensity (2.1) can be presented as multidimensional vector $\vec{A}=\left\{a_{n}^{m}, b_{n}^{m}\right\}$ with infinity number of components $(0 \leq m \leq n<\infty)$. Then we can determine an receiving vector $\vec{Z}$ (which is also infinite dimensional) so that the intensity $J$ registered by the detector is equal to the scalar multiplication

$$
J=\vec{A} \cdot \vec{Z}
$$

From (2.1) and (2.2) it follows that:

$\vec{R}=\left\{x_{n}^{m}, y_{n}^{m}\right\}$, where $(0 \leq m \leq n<\infty)$,

$x_{n}^{m}=\operatorname{cosm} \varphi P_{n}^{m}(\sin \theta)$,

$y_{n}^{m}=\operatorname{sinm} \varphi P_{n}^{m}(\sin \theta)$.

The vectors $\vec{A}$ and $\vec{R}$ can be represented in the complex form as:

$\vec{A}=\left\{r_{n}^{m}\right\} \quad r_{n}^{m}=a_{n}^{m}+i b_{n}^{m}$

$\vec{R}=\left\{Z_{n}^{m}\right\} \quad Z_{n}^{m}=x_{n}^{m}+i y_{n}^{m}=e^{i m \varphi} P_{n}^{m}(\sin \theta)$

and in the case of vectors with complex components

$$
J=\frac{1}{2}\left(\vec{A} \cdot \vec{R}^{*}+\vec{A}^{*} \cdot \vec{R}\right)
$$

Thus, at the Earth, because of its rotation, the diurnal variation would be registered. This variation is determined by the Fourier series

$$
J(t)=\sum_{n=0}^{\infty}\left(A_{m} \cos m \omega t+B_{m} \sin m \omega t\right)
$$

where $A_{m}=\sum_{n=m}^{\infty}\left(a_{n}^{m} x_{n}^{m}+b_{n}^{m} y_{n}^{m}\right), B_{m}=\sum_{n=m}^{\infty}\left(-a_{n}^{m} y_{n}^{m}+b_{n}^{m} x_{n}^{m}\right), \omega$ is the angular velocity of Earth's rotation.

A calculation of receiving vectors $\vec{Z}$ is made taking into account the directional sensitivity of the detector, particle drift angles, coupling coefficients and power spectrum of anisotropy.

The devices with different receiving vectors register corresponding intensity $J$ which is determined as follows:

$$
J=\sum_{n=0}^{\infty} \sum_{m=0}^{n}\left(a_{n}^{m} x_{n}^{m}+b_{n}^{m} y_{n}^{m}\right),
$$

If the number of devices is enough then the components $a_{n}^{m}, b_{n}^{m}$ of the vector of cosmic ray distribution intensity (for the first two spherical harmonics) at each time moment can be found from the presented system of equations (2.5).

Since the power sensitivity of the detectors are different, it is necessary to introduce the quantities $k_{n}^{(j)}$ defined by

$$
k_{n}^{(j)}=\frac{\int_{\varepsilon_{\min }}^{\infty} W^{j}(\varepsilon) f_{n}(\varepsilon) d \varepsilon}{\int_{\varepsilon_{\min }}^{\infty} W^{0}(\varepsilon) f_{n}(\varepsilon) d \varepsilon},
$$


where $f_{n}(\varepsilon)$ is the power spectrum of the spherical harmonic of the order $n, W^{j}(\varepsilon)(j=1,2, \ldots)$ are the differential energy sensitivities if the detector in consideration, and $W^{0}$ is some arbitrary differential sensitivity. Equation (2.5) assumes the form

$$
I^{j}=\sum_{n=0}^{\infty} \sum_{m=0}^{n}\left(a_{n}^{m, 0} x_{n}^{m, j}+b_{n}^{m, 0} y_{n}^{m, j}\right) k_{n}^{j}
$$

where $a_{n}^{m, 0}$ and $b_{n}^{m, 0}$ are unknown coefficients independent of $j$. The system obtained can be solved by means of the least squares method provided that the series converges quickly and higher order terms may be neglected.

In order to get free from noise due to the instability of the detectors the next original data filtering method was used. The observed intensity $J$ is divided into high frequency $J_{h . f}$. and low frequency $J_{l . f}$. parts and thus the device variations will be in the first part, the anisotropy in the second part and the isotropic variations in both parts. The low frequency part is revealed by the moving average method over 37 hours. So, the high frequency is equal to $J_{h . f}=J-J_{l . f}$. Having analyzed the high frequency part by means of equation (2.7), we obtain the components of anisotropy vector $\vec{A}$.
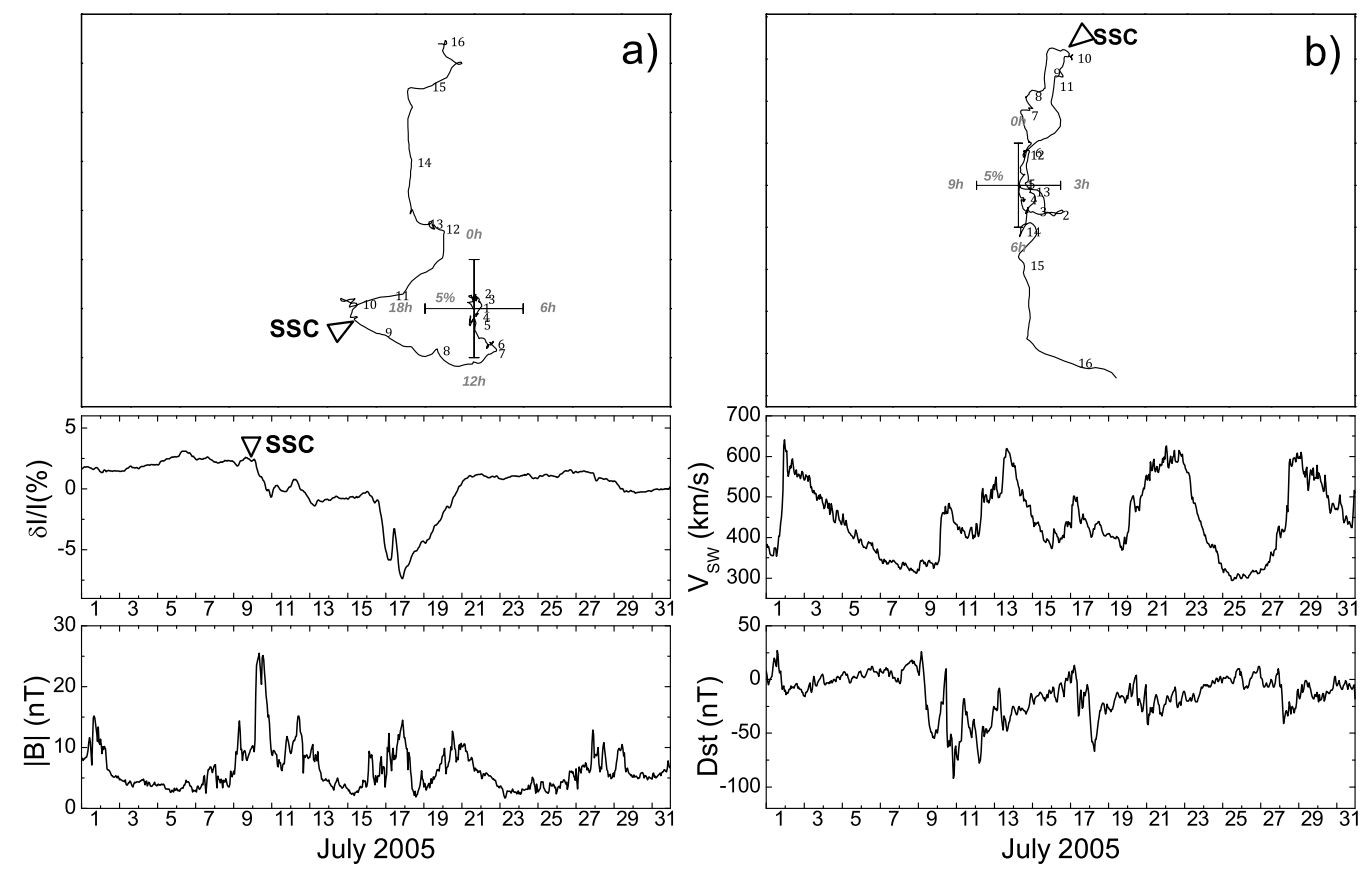

Figure 1: The vector diagram of the components of tensor anisotropy $R_{2}^{1}$ (a), $R_{2}^{2}$ (b) in July 2005. On a hour dial the numbers indicates the day of month. $\delta I / I$ is the isotropic part of cosmic ray intensity, $V_{S W}$ is the solar wind speed, $|B|$ is the magnitude of interplanetary magnetic field intensity, the Dst is the index of geomagnetic activity.

\section{Obtained results}

We have obtained the behavior of diurnal $\left(R_{1}^{1}\right)$, antisymmmetric diurnal $\left(R_{2}^{1}\right)$ and semidiurnal $\left(R_{2}^{2}\right)$ variations of cosmic ray intensity by the method of global survey using the hourly data 

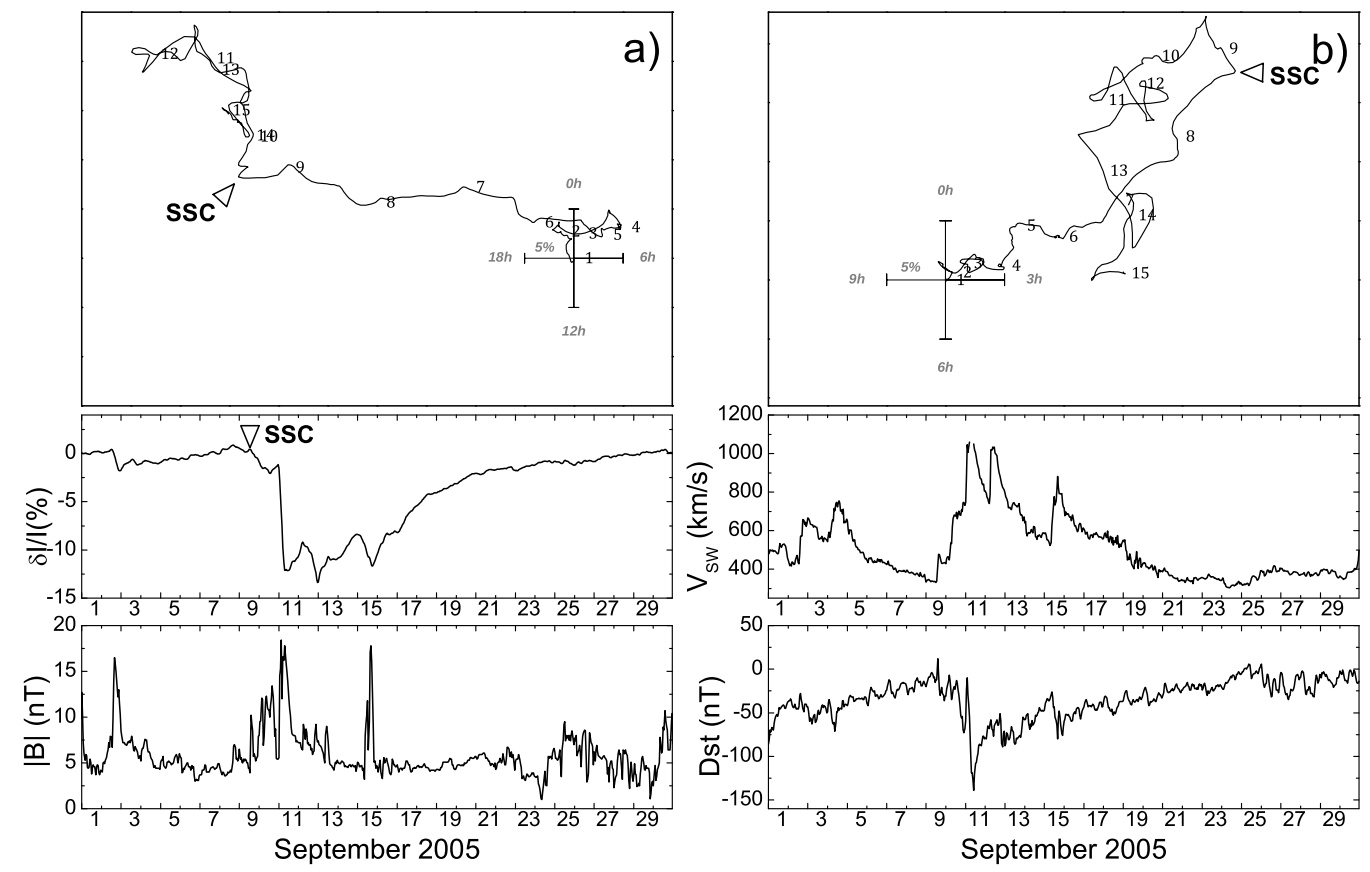

Figure 2: The vector diagram of the components of tensor anisotropy $R_{2}^{1}$ (a), $R_{2}^{2}$ (b) in September 2005. On a hour dial the numbers indicates the day of month. $\delta I / I$ is the isotropic part of cosmic ray intensity, $V_{S W}$ is the solar wind speed, $|B|$ is the magnitude of interplanetary magnetic field intensity, the Dst is the index of geomagnetic activity.

of the neutron monitor worldwide network ( $\sim 30$ stations) for 1990-2012. Also the data about the interplanetary magnetic field state, solar wind speed, Dst-index and computed coronal field synoptic charts of the Wilcox Solar Observatory and some solar proton events affecting the earth environment ${ }^{6}$. The analysis of the obtained data shows the unusual amplitude-phase oscillations of antisymmetric diurnal $R_{2}^{1}$ and semidiurnal $R_{2}^{2}$ variations (table 1 ). The next 2 events of unusual behavior of the tensor anisotropy components during Forbush decreases at July 2005 (fig.1) and September 2005 (fig.2) are shown as examples. The SSC beginnings are shown by arrows.

In September 2005 the decrease of the cosmic ray intensity has an amplitude of 14\%. The reason of this effect is the solar flare that happened in 07.09.2005 on the eastern limb (S06E98) of the Sun at the south of helioequator. This decrease is caused by the increase of interplanetary magnetic field in 09.09.2005 and accompanied by the solar wind speed increase up to $1200 \mathrm{~km} / \mathrm{s}$ and the geomagnetic disturbance is caused. Before the beginning of forbush decrease the component $R_{2}^{1}$ was directed to $\sim 18 \mathrm{~h}$ and $R_{2}^{2}$ to $\sim 2 \mathrm{~h}$ of local time. Hence, with the arrival of shock wave both vectors $R_{2}^{1}$ and $R_{2}^{2}$ changed their to $\sim 23 \mathrm{~h}$ and $\sim 8 \mathrm{~h}$ respectively.

In July 2005 the forbush decreases at 10th days of month with amplitude $\sim 3 \%$ is observed. As in the previous event, this decreases were accompanied by the jumps of solar wind speed up to $500 \mathrm{~km} / \mathrm{s}$, the interplanetary magnetic field intensity and Dst-index

The possible reasons of this abrupt direction changes of $R_{2}^{1}$ and $R_{2}^{2}$ to the reverse are the

\footnotetext{
${ }^{6} \mathrm{http}: / /$ umbra.nascom.nasa.gov/SEP/seps.html
} 
Table 1: The selected months when the components $R_{2}^{1}$ and $R_{2}^{2}$ have the unusual behavior

\begin{tabular}{|c|c|c|c|}
\hline No & Date & No & Date \\
\hline 1 & November 1990 & 20 & June 2005 \\
\hline 2 & December 1991 & 21 & July 2005 \\
\hline 3 & May 1992 & 22 & August 2005 \\
\hline 4 & September 1992 & 23 & September 2005 \\
\hline 5 & October 1993 & 24 & July 2007 \\
\hline 6 & November 1993 & 25 & September 2007 \\
\hline 7 & January 1994 & 26 & February 2010 \\
\hline 8 & April 1995 & 27 & March 2010 \\
\hline 9 & April 1996 & 28 & September 2010 \\
\hline 10 & March 2000 & 29 & November 2010 \\
\hline 11 & October 2000 & 30 & December 2010 \\
\hline 12 & January 2001 & 31 & February 2011 \\
\hline 13 & August 2001 & 32 & March 2011 \\
\hline 14 & September 2002 & 33 & September 2011 \\
\hline 15 & October 2002 & 34 & March 2012 \\
\hline 16 & March 2003 & 35 & April 2012 \\
\hline 17 & June 2003 & 36 & July 2012 \\
\hline 18 & July 2003 & 37 & September 2012 \\
\hline 19 & August 2003 & 38 & November 2012 \\
\hline
\end{tabular}

formation of a magnetic mirror at the jumps of interplanetary magnetic field intensity. Inside of the regular magnetic field the trapped particles changes are occur. The similar events during forbush decrease or solar wind speed abrupt increases are presented in the table.

\section{Conclusion}

The behavior of the components $R_{1}^{1}, R_{2}^{1}$ and $R_{2}^{2}$ during the various short-term solar wind disturbances obtained using the method of global survey by the hourly data of neutron monitor worldwide network. The unusual change of amplitude phase oscillations of anisymmetric diurnal and semidiurnal variations of cosmic ray intensity, specifically the abrupt change of the direction of vectors $R_{2}^{1}$ and $R_{2}^{2}$. The possible reason of this phenomenon is the formation of the magnetic mirror at the jumps of interplanetary magnetic field intensity.

\section{Acknowledgments}

The work has been supported by the Russian Fund for Basic Research (grant nos. 13-0200989-a, 15-42-05085 r_vostok-a, 15-02-03299 r_vostok-a), the Program of the Presidium of RAS 
No.31 and the grant of the President of Russian Federation on the state support of leading scientific schools (NSh-3269.2014.2).

\section{References}

[1] G.F. Krymsky, A.I. Kuzmin, P.A. Krivoshapkin et al., Cosmic rays and solar wind (in Russian), Nauka, Novosibirsk (1981) 224

[2] G.V. Skreipin, P.A. Krivoshapkin, G.F. Krymsky and A.I. Kuzmin, Acceleration of charged particles in shear flow, Pis'ma v JETP (in Russian) 33 (1981) 416

[3] E.G. Berezhko, On acceleration possibility of charged particles under cosmic plasma shearing flows, Proc. 17th Internat. Cosmic Ray Conf 3 (1981) 506

[4] E.G. Berezhko and G.F. Krymsky, Kinetic consideration of charged particle acceleration process in collisionless plasma shear flow, Soviet Astronomy Letters (in Russian) 7 (1981) 352

[5] A.M. Altukhov, G.F. Krymsky, A.I. Kuzmin, The method of "global survey”for investigating cosmic ray modulation, Proc. 11-th Int. Conf. on Cosmic Rays 4 (1970) 457

[6] G. F. Krymsky, P. A. Krivoshapkin, V. P. Mamrukova, S. K. Gerasimova, Study of cosmic ray semidiurnal variations, Journal of Physics: Conference Series 409 (2013) 012174

[7] G.K. Gerasimova, P.Yu. Gololobov, P.A. Krivoshapkin, G.F. Krymsky, Studying semidiurnal variations in galactic cosmic Rays, Bulletin of the Russian Academy of Sciences. Physics 77 (2013) 526

[8] G.F. Krymsky, P.A. Krivoshapkin, S.K. Gerasimova, P.Yu. Gololobov, Tensor anisotropy of cosmic Rays, Astronomy Letters 40 (2014) 230 